Mathematical Modelling and Analysis

Volume 20 Number 6, November 2015, 782-801

http://dx.doi.org/10.3846/13926292.2015.1113206

(c) Vilnius Gediminas Technical University, 2015
Publisher: Taylor\&Francis and VGTU

http://www.tandfonline.com/TMMA

ISSN: $1392-6292$

eISSN: $1648-3510$

\title{
Bifurcation Analysis in a Delay Differential Equations, which Confers a Strong Allee Effect in Escherichia Coli
}

\section{Qiubao Wang and Ruilan Tian}

\author{
Department of Mathematics and Physics, Shijiazhuang Tiedao University \\ 050043 Shijiazhuang, PR China \\ E-mail: wangqiubao12@sina.com
}

Received April 16, 2015; revised October 11, 2015; published online November 15, 2015

\begin{abstract}
The paper addresses the bifurcations for a delay differential model with parameters which confers a strong Allee effect in Escherichia coli. Stability and local Hopf bifurcations are analyzed when the delay $\tau$ or $\sigma$ as parameter. It is also found that there is a non-resonant double Hopf bifurcation occur due to the vanishing of the real parts of two pairs of characteristic roots. We transform the original system into a finite dimensional system by the center manifold theory and simplify the system further by the normal form method. Then, we obtain a complete bifurcation diagram of the system. Finally, we provide numerical results to illustrate our conclusions. There are many interesting phenomena, such as attractive quasi-periodic solution and three-dimensional invariant torus.
\end{abstract}

Keywords: double Hopf, bifurcation, delay, center manifold.

AMS Subject Classification: $34 \mathrm{H} 20$.

\section{Introduction}

Allee was initially stimulated by an example only loosely linked to the current interpretation of the Allee effect: he showed that goldfish grew faster in water which had previously contained other goldfish, than in water that had not (see [1]). Allee saw these phenomena as "automatic cooperation", believing that the beneficial effects of numbers of animals present in a population represented a fundamental biological principle. By 1953, E. P. Odum was referring to "Allee's principle" as the concept that "undercrowding (or lack of aggregation) may be limiting" (see [16]). Reduction in fitness or population growth at low abundance has received considerable attention in conservation genetics, under such guises as the "50/500 rule" (see [21]), and is also widely debated in fisheries science, where it is usually referred to as depensation (see [15]). Depensation is principally a population level phenomenon, which may or may not 
arise from changes in individual fitness, and thus need not be directly analogous to the Allee effect.

Although the Allee effect is reasonably well known, the concept has a range of meanings, not all of which are acknowledged by contemporary use. Allee did not provide a definition but he clearly considered "certain aspects of survival values" rather than total fitness. Until 1999, Stephens P. A. (see [22]) defined the Allee effect as: a positive relationship between any component of individual fitness and either numbers or density of conspecifics. After that, the Allee effect be thought as a phenomenon in biology characterized by a correlation between population size or density and the mean individual fitness of a population or species (see $[3,5,18,20]$ ). Differential equation is a very powerful tool to explore problems, so a lot of differential equations models about Allee effect appear. Holmes E.E.(see [10]) studied a partial differential equations(PDE) in ecological which add Allee effect. Čiegis R. and Bugajev A. (see [4]) analysed a model of bacterial self-organization and gave the specific numerical approximation. Painter K.J. and Hillen T. (see [17]) obtained spatio-temporal chaos in a chemotaxis model. The stability analysis of such systems is a very important topic in theoretical and applied mathematics. For a long time, it has been recognized that delays can have very complicated impact on the system (see $[2,19,23,29])$. Yan J. (see [29]) discussed an impulsive delay differential equation with Allee effect, and obtained a periodic solution and its stability. Lingchong You, Robert Smith Randy et al. (for details, see [20]) engineered a gene circuit to confer a strong Allee effect in Escherichia coli and examined its impact on spread and survival. They used the LuxR/LuxI quorum-sensing (QS) system from Vibrio fischeri (see [14]) and the CcdA/CcdB toxinCantitoxin module to control population survival. The detailed operational principle of they circuit was described in [20], so we will leave it at that. Their circuit can be modeled by the following delayed differential equations:

$$
\left\{\begin{aligned}
\frac{d C}{d t} & =\mu C(1-C)-\frac{\gamma C}{\beta+[A(t-\tau)]}, \\
\frac{d[A]}{d t} & =k_{A} C-k_{d A}[A]
\end{aligned}\right.
$$

where $C$ and $[A]$ represent the bacterial density and the concentration of AHL $(\mu M)$,respectively. $\mu$ represents the maximum specific growth rate $\left(h^{-1}\right), k_{A}$ represents the synthesis rate constant of $\mathrm{AHL}\left(\mu M h^{-1}\right), k_{d A}$ represents the degradation rate constant of AHL $\left(h^{-1}\right), \tau$ represents the time delay of the activation of gene expression by the LuxRAHL complex $(h), t$ represents simulation time $(h), \gamma$ is a lumped term that represents the killing rate of CcdB $\left(\mu M h^{-1}\right)$, and $\beta$ is a lumped term that represents the amount of CcdA leading to half-maximal killing rate of $\mathrm{CcdB}(\mu M)$.

Three cases were considered, with the circuit $\mathrm{OFF}, \mathrm{ON}$ and $\mathrm{ON}+$ rescue. They declared that their protocol may be amenable for the study of Allee effects in natural systems, including Drosophila melanogaster and One could dispersed an established population of flies to new medium in a separate culture and examine reproductive success.

But in the whole paper, they did not discuss the effects of the time delay $\tau$ 
on the bacterial density. In our recent works [24,25], we discussed the stability and the Hopf bifurcation of some models with delay-dependent parameters. Moreover, it is well known that the delayed logistic differential equation

$$
\frac{d x}{d t}=r x\left(1-\frac{x(t-\tau)}{k}\right)
$$

is used to model the evolution of a single species $x(t)$ (see $[11,12,30])$. Along this line, we established a new model as follows:

$$
\left\{\begin{aligned}
\frac{d C}{d t} & =\mu C\left(1-\frac{C(t-\sigma)}{K}\right)-\frac{\gamma C}{\beta+[A(t-\tau)]}, \\
\frac{d[A]}{d t} & =k_{A} C-k_{d A}[A],
\end{aligned}\right.
$$

where $\sigma$ represents the time of bacterial split $(h), K$ represents the maximum capacity of container for bacterial. In our paper, we investigate the dynamics near the equilibriums and hope to derive the perfect theoretical results. In fact, we not only discuss the Hopf bifurcation when $\tau$ or $\sigma$ as a parameter, but also consider a codimension-2 bifurcation on a two-parameter plane, i.e. double Hopf bifurcation. For double Hopf bifurcation, we usually can observe lots of interesting and complicated dynamic behavior. Especially, strong resonant double Hopf bifurcation can present more complex nonlinear behavior because it may be a codimension-3 bifurcation.

In order to get a deeper insight into the double Hopf bifurcation analysis, we may apply and extend the methods and results in $[6,7]$ to the case of two bifurcation parameters, and obtain the normal forms for double Hopf bifurcation without strong resonance. For strong resonant case, we will consider in another paper.

The rest of this paper is organized as follows. In Section 2, we discuss nonnegativeness and boundedness of solutions for the system (1.1). In Section 3, we consider the stability and the local Hopf bifurcation of the equilibriums when the time delay $\sigma$ and $\tau$ as parameters respectively. In Section 4 , the existences of double Hopf bifurcation is discussed. The normal form method and the center manifold theory are used to analyze the double Hopf bifurcation for system. In Section 5, we give some numerical examples to illustrate our results at the previous sections. In Section 6, we summarize our results.

\section{Nonnegativeness and boundedness of solutions}

We denote by $X=C\left([-\kappa, 0], R_{+}^{2}\right)$ the Banach space of continuous functions mapping the internal $[-\kappa, 0]$ into $R_{+}^{2}$ equipped with the sup-norm, where $\kappa=$ $\max (\sigma, \tau)$. By the standard theory of functional differential equations, we know that for any $\phi \in X$ there exists a unique solution $T(t, \phi)=(C(t, \phi),[A(t, \phi)])$ of the system (1.1), where $T_{0}=\phi$. The initial conditions are given by $C(\theta)=$ $\phi_{1}(\theta),[A(\theta)]=\phi_{2}(\theta), \theta \in[-\kappa, 0]$, where $\phi=\left(\phi_{1}, \phi_{2}\right) \in X$ with $\phi_{1} \geq 0, \phi_{2} \geq 0$.

Theorem 1. Solution of system (1.1) corresponding to the above initial conditions remains nonnegative and ultimately bounded for all $t \geq 0$. 
Proof. Nonnegative: The proof of nonnegative for $C(t)$ is similar to that of Lemma 2.1 of [28], and thus details are omitted here for the sake of brevity. For $[A(t)]$, we assume the contrary, and let $\tilde{t}>0$ be the first time such that $[A(\widetilde{t})]=0$, then by the second equation of system (1.1), we have $[A(\widetilde{t})]^{\prime}=$ $k_{A} C(\widetilde{t}) \geq 0$. If $C(\widetilde{t})=0$, then $C(t)=0,[A(t)]=0$ for all $t \geq \widetilde{t}$. We omit this trivial case. Hence, $[A(t)]<0$ for $t \in(\widetilde{t}-\epsilon, \widetilde{t})$, where $\epsilon>0$ is sufficiently small, which contradicts $[A(t)]>0$ for $t<\tilde{t}$. Ultimately bounded: From the first equation of (1.1), we obtain

$$
\frac{d C}{d t} \leq \mu C
$$

Hence, for $t>\tau$ we get that $C(t) \leq C(t-\tau) e^{\mu \tau}$, i.e.,

$$
C(t) e^{-\mu \tau} \leq C(t-\tau) .
$$

On substituting the above into (1.1), we obtain

$$
\frac{d C(t)}{d t} \leq \mu C\left(1-\frac{C e^{-\mu \tau}}{K}\right), t>\tau,
$$

which clearly implies that,

$$
\lim \sup _{t \rightarrow \infty} C(t) \leq e^{\mu \tau} K
$$

For $[A(t)]$, we know that $\frac{d[A(t)]}{d t} \leq k_{A} K e^{\mu \tau}-k_{d A}[A(t)]$, for $t>\tau$, so

$$
\lim \sup _{t \rightarrow \infty}[A(t)] \leq \frac{k_{A} K e^{\mu \tau}}{k_{d A}}
$$

We complete the proof.

\section{Stability of the equilibrium and existence of the Hopf bifurcation}

For the system (1.1), it is obvious that it has a trivial fixed point $E_{0}(0,0)$ and two possible fixed points $E_{1}\left(\frac{k_{d A}}{k_{A}} A_{-}, A_{-}\right), E_{2}\left(\frac{k_{d A}}{k_{A}} A_{+}, A_{+}\right)$, where $A_{\mp}$ are the real roots of

$$
\mu k_{d A} A^{2}+\left(\mu \beta k_{d A}-\mu K k_{A}\right) A+\gamma K k_{A}-\mu \beta K k_{A}=0 .
$$

The characteristic equation of its corresponding linear system around the $E_{0}$ is given by

$$
D(\lambda)=\lambda^{2}+\left(k_{d A}-\mu+\frac{\gamma}{\beta}\right) \lambda+k_{d A}\left(\frac{\gamma}{\beta}-\mu\right)=0 .
$$

Obviously, if $\frac{\gamma}{\beta}>\mu$, then $E_{0}$ is stable for all $\sigma \geq 0, \tau \geq 0$. If $\frac{\gamma}{\beta}<\mu$, then $E_{0}(0,0)$ is unstable for all $\sigma \geq 0, \tau \geq 0$. By the translation $u_{1}(t)=$ 
$C(t)-\frac{k_{d A}}{k_{A}} A^{*}, u_{2}(t)=[A(t)]-A^{*}$, where $A^{*}$ is one of $A_{ \pm}$, then system (1.1) is equivalent to

$$
\left\{\begin{aligned}
\frac{d u_{1}}{d t} & =\mu\left(u_{1}+\frac{k_{d A}}{k_{A}} A^{*}\right)\left(1-\frac{u_{1}(t-\sigma)+\frac{k_{d A}}{k_{A}} A^{*}}{K}\right)-\frac{\gamma\left(u_{1}+\frac{k_{d A}}{k_{A}} A^{*}\right)}{\beta+u_{2}(t-\tau)+A^{*}}, \\
\frac{d u_{2}}{d t} & =k_{A}\left(u_{1}+\frac{k_{d A}}{k_{A}} A^{*}\right)-k_{d A}\left(u_{2}+A^{*}\right) .
\end{aligned}\right.
$$

The linearization of $(3.1)$ at $\left(u_{1}, u_{2}\right)=(0,0)$ is

$$
\left\{\begin{array}{l}
\frac{d u_{1}}{d t}=-\frac{\mu \frac{k_{d A}}{k_{A}} A^{*}}{K} u_{1}(t-\sigma)+\frac{\gamma \frac{k_{d A}}{k_{A}} A^{*}}{\left(\beta+A^{*}\right)^{2}} u_{2}(t-\tau), \\
\frac{d u_{2}}{d t}=k_{A} u_{1}-k_{d A} u_{2}
\end{array}\right.
$$

Thus, the characteristic equation of (3.2) is

$$
D(\lambda)=\lambda^{2}+k_{d A} \lambda+a\left(\lambda+k_{d A}\right) e^{-\lambda \sigma}-b e^{-\lambda \tau}=0,
$$

where $a=\frac{\mu \frac{k_{d A}}{k_{A}} A^{*}}{K}, b=\frac{\gamma k_{d A} A^{*}}{\left(\beta+A^{*}\right)^{2}}$. We can obtain the following results about the fixed point bifurcation.

Theorem 2. If $K k_{A}=k_{d A} \beta$, the system (1.1) undergoes a pitchfork bifurcation at $\mu=\frac{\gamma}{\beta}$.

Proof. By the hypothesis $K k_{A}=k_{d A} \beta$, when $\mu=\frac{\gamma}{\beta}$, the (3.3) with $\sigma=\tau=0$ has a zero root and another negative root. Note that, if $\mu \leq \frac{\gamma}{\beta}$, then there is only equilibrium $E_{0}$, it is stable for $\mu \leq \frac{\gamma}{\beta}$ and unstable for $\mu>\frac{\gamma}{\beta}$. At the same time, on the side of $\mu>\frac{\gamma}{\beta}$, two new stable equilibria are created and given by $E_{1}, E_{2}$. Therefore the system (1.1) undergoes a pitchfork bifurcation at $\mu=\frac{\gamma}{\beta}$.

Here, we further explored the system for bifurcation analysis. This enabled us to find the system also undergoes a Hopf bifurcation for some $\sigma$ or $\tau$. By the above theorem, the system maybe undergoes a high co-dimensional bifurcations, i.e., Hopf-pitchfork bifurcation. For this bifurcation, the system may exhibit chaos via period-doubling bifurcations as the unfolding parameter values are far away from the critical point. That is, you can find the existence of highly irregular and chaotic-like dynamics in the system. We will discuss this situation in the next paper. In this paper, we only focus on Hopf and Double Hopf bifurcation.

In the rest of this section, we show the Hopf bifurcation of the system when the time delay is used as parameter.

\subsection{Case $\sigma=0$}

We first consider the situation with no delay $\sigma$. If $i \omega(\omega>0)$ is a root of (3.3) then

$$
-\omega^{2}+i \omega\left(a+K_{d A}\right)+a k_{d A}-b e^{-i \omega \tau}=0 .
$$


Separating the real and imaginary parts, we have

$$
\left\{\begin{aligned}
-\omega^{2}+a k_{d A}-b \cos \omega \tau & =0 \\
\omega\left(a+K_{d A}\right)+b \sin \omega \tau & =0
\end{aligned}\right.
$$

which leads to

$$
\omega^{4}+\left(a^{2}+k_{d A}^{2}\right) \omega^{2}+a^{2} k_{d A}^{2}-b^{2}=0 .
$$

Denote $b-a k_{d A}=H$. It is easily to see that if either $H>0$, then (3.5) has a unique positive root

$$
\omega_{0}^{2}=\frac{1}{2}\left[-\left(a^{2}+k_{d A}^{2}\right)+\sqrt{\left(a^{2}-k^{2}\right)^{2}+4 b^{2}}\right] .
$$

From (3.4), we find

$$
\tau_{j}=\frac{1}{\omega_{0}}\left[\pi-\arcsin \left(a+k_{d A}\right) \omega_{0}+2 j \pi\right], j=0,1, \cdots .
$$

Denote $\lambda(\tau)=\alpha(\tau)+i \omega(\tau)$ the root of $(3.3)$ such that $\alpha\left(\tau_{j}\right)=0, \omega\left(\tau_{j}\right)=\omega_{0}$. Substituting $\lambda(\tau)$ into (3.3) and taking the derivative with respect to $\tau$, we have

$$
\left[\frac{d \lambda}{d \tau}\right]^{-1}=\frac{2 \lambda+k_{d A}+a}{-b \lambda e^{-\lambda \tau}}-\frac{\tau}{\lambda},
$$

which, together with (3.4), leads to

$$
\begin{aligned}
\operatorname{Re} & {\left[\frac{d \lambda}{d \tau}\right]_{\tau=\tau_{j}}^{-1}=\frac{2 \lambda+k_{d A}+a}{-b \lambda e^{-\lambda \tau}} } \\
& =\operatorname{Re}\left[\frac{2 i \omega+k_{d A}+a}{-i \omega\left(a k_{d A}-\omega^{2}+i \omega\left(k_{d A}+a\right)\right)}\right]=\frac{\left(k_{d A}-a\right)^{2}+2 \omega^{2}}{\left(a k_{d A}-\omega^{2}\right)^{2}+\left(k_{d A}+a\right)^{2} \omega^{2}} .
\end{aligned}
$$

So we have

$$
\operatorname{Re}\left[\frac{d \lambda}{d \tau}\right]_{\tau=\tau_{j}}^{-1}>0 .
$$

Notice that when $\tau=0$, the equation (3.3) becomes

$$
\lambda^{2}+\left(a+k_{d A}\right) \lambda+a k_{d A}-b=0 .
$$

If $b<a k_{d A}$, then the equation (3.8) has two roots with negative real parts. Otherwise, if $b>a k_{d A}$, there is at least one root with positive real parts. Therefore, we can obtain the following lemma.

Lemma 1. When $\sigma=0$. Let $\tau_{j}(j=0,1, \ldots)$ be defined by $(3.6)$.

1. If $H<0$, then the equation (3.3) has no root with positive real parts for all $\tau \geq 0$. 
2. If $H>0$, then there exits $\tau_{j}$, such that the equation (3.3) has a pair of purely imaginary roots $\pm i \omega_{0}$ when $\tau=\tau_{j}$.

From the above lemma and (3.7) and the Hopf bifurcation theorem for functional differential equaitons (Theorem 1.1 in Chapter 10 of Hale and Lunel [8]), we have the following results on stability and bifurcation to system (1.1).

Theorem 3. For system (3.1), when $\sigma=0$

1. If $H<0$, then the equilibrium $(0,0)$ is asymptotically stable for all $\tau \geq 0$.

2. If $H>0$, then the equilibrium $(0,0)$ is unstable for all $\tau \geq 0$, and the system (3.1) undergoes a Hopf bifurcation at $(0,0)$ when $\tau=\tau_{j},(j=0,1, \cdots)$.

Remark 1. When $\tau=0$, the model is the same as [20]. But in [20], there is a lot of directions about the experimental phenomena, but no theoretical analysis. In this paper, we analysis the dynamics behavior of the model in detailed, and give the reasons which cause the experimental phenomena from a mathematical point.

\subsection{Case $\tau=0$}

Next, we consider the another situation, when $\tau=0, \sigma \geq 0$. Thus, the characteristic equation of (3.2) becomes

$$
D(\lambda)=\lambda^{2}+k_{d A} \lambda+a\left(\lambda+k_{d A}\right) e^{-\lambda \sigma}-b=0 .
$$

For convenience, we make the following assumption $\left(H_{1}\right): a^{2}-k_{d A}^{2}-2 b>0$ and denote $\Delta=\left(2 b+k_{d A}^{2}-a^{2}\right)^{2}-4\left(b^{2}-a^{2} k_{d A}^{2}\right)$. Using the similarly deduction, one can easily get the following conclusions.

Lemma 2. For equation (3.9):

1. If $H<0$, then there exits $\sigma_{j}$, such that the equation (3.9) has a pair of purely imaginary roots $\pm i \omega_{0}$ when $\sigma=\sigma_{j}$;

2. If $H>0$ and $\left(H_{1}\right)$ is not satisfied, or $H>0, \Delta<0$ and $\left(H_{1}\right)$ is satisfied, then the equation (3.9) has at least one root with positive real part for all $\sigma \geq 0$

3. If $H>0, \Delta=0$ and $\left(H_{1}\right)$ is satisfied, then there exits $\hat{\sigma}_{j}$, such that the equation (3.9) has a pair of purely imaginary roots $\pm i \hat{\omega}_{0}$ when $\sigma=\hat{\sigma}_{j}$;

4. If $H>0, \Delta>0$ and $\left(H_{1}\right)$ is satisfied, then there exits $\sigma_{j}^{ \pm}$, such that the equation (3.9) has a pair of purely imaginary roots $\pm i \omega_{ \pm}$when $\sigma=\sigma_{j}^{ \pm}$, respectively,

where

$$
\begin{aligned}
& \omega_{0}=\frac{1}{\sqrt{2}}\left[a^{2}-2 b-k_{d A}^{2}+\sqrt{\Delta}\right]^{\frac{1}{2}}, \quad \sigma_{j}=\frac{1}{\omega_{0}}\left[\arccos \frac{k_{d A} b}{a\left(\omega_{0}^{2}+k_{d A}^{2}\right)}+2 j \pi\right] ; \\
& \hat{\omega}_{0}=\frac{1}{\sqrt{2}}\left(a^{2}-2 b-k_{d A}^{2}\right)^{\frac{1}{2}}, \quad \hat{\sigma}_{j}=\frac{1}{\hat{\omega}_{0}}\left[\arccos \frac{k_{d A} b}{a\left(\hat{\omega}_{0}^{2}+k_{d A}^{2}\right)}+2 j \pi\right] ; \\
& \omega_{ \pm}=\frac{1}{\sqrt{2}}\left[a^{2}-2 b-k_{d A}^{2} \pm \sqrt{\Delta}\right]^{\frac{1}{2}}, \quad \sigma_{j}^{ \pm}=\frac{1}{\omega_{ \pm}}\left[\arccos \frac{k_{d A} b}{a\left(\omega_{ \pm}^{2}+k_{d A}^{2}\right)}+2 j \pi\right] .
\end{aligned}
$$


Let $\lambda=\alpha(\sigma)+i \omega(\sigma)$ is the root of (3.9) such that $\alpha(\sigma)=0, \omega(\sigma)>0$ for the above critical values. Substituting $\lambda(\sigma)$ into (3.9) and taking the derivative with respect to $\sigma$, we have the following conclusions easily.

Lemma 3. $\alpha^{\prime}\left(\sigma_{j}\right)>0, \alpha^{\prime}\left(\sigma_{j}^{+}\right)>0, \alpha^{\prime}\left(\sigma_{j}^{-}\right)<0$ and $\alpha^{\prime}\left(\hat{\sigma}_{j}\right)=0$.

Proof. By direct calculation, one can easily obtain that

$$
R e\left[\frac{d \lambda}{d \sigma}\right]_{\sigma=\sigma_{j}}^{-1}=\frac{2 b+2 \omega_{0}^{2}+k_{d A}^{2}-a^{2}}{a^{2}\left(k_{d A}^{2}+\omega_{0}^{2}\right)} .
$$

Note that $\omega_{0}=\frac{1}{\sqrt{2}}\left[a^{2}-2 b-k_{d A}^{2}+\sqrt{\Delta}\right]^{\frac{1}{2}}$. Therefore, $\operatorname{Re}\left[\frac{d \lambda}{d \sigma}\right]_{\sigma=\sigma_{j}}^{-1}=\frac{\sqrt{\Delta}}{a^{2}\left(k_{d A}^{2}+\omega_{0}^{2}\right)}>$ 0 , it follows that $\alpha^{\prime}\left(\sigma_{j}\right)>0$. The proof of that three conclusions is similarly.

Hence, there is the following conclusions.

Theorem 4. For system (3.1), when $\tau=0$ :

1. If $H<0$, then the system (3.1) undergoes a Hopf bifurcation at the origin $(0,0)$ when $\sigma=\sigma_{j}, j=0,1, \ldots$ The zero solution is asymptotically stable for $\sigma \in\left[0, \sigma_{0}\right)$ and unstable for $\sigma>\sigma_{0}$;

2. If $H>0$ and $\left(H_{1}\right)$ is not satisfied, or $H>0, \Delta<0$ and $\left(H_{1}\right)$ is satisfied, then the zero solution is unstable for all $\sigma \geq 0$;

3. If $H>0, \Delta=0$ and $\left(H_{1}\right)$ is satisfied, then the bifurcation is degenerated;

4. If $H>0, \Delta>0$ and $\left(H_{1}\right)$ is satisfied, then the system (3.1) undergoes a Hopf bifurcation at the origin $(0,0)$ when $\sigma=\sigma_{j}^{ \pm}, j=0,1, \ldots$.

Moreover, if $\sigma_{0}^{+}>\sigma_{0}^{-}$, there exists an integer $m \geq 0$ such that the zero solution is unstable when $\sigma \in\left(\sigma_{j-1}^{+}, \sigma_{j}^{-}\right)$for $j=0,1, \ldots, m$ and $\sigma>\sigma_{m}^{+}$, and asymptotically stable when $\sigma \in\left(\sigma_{j}^{-}, \sigma_{j}^{+}\right)$for $j=0,1, \ldots, m$, where $\sigma_{-1}^{+}=0$.

Remark 2. Notice that, when $\tau=0$, our model become a logistic model. In comparison with a classical logistic model, there is another negative term in the right of first equation. In the next section, we will discuss a codim- 2 bifurcation, double Hopf bifurcation, which does not appearance in classical logistic model in general.

Remark 3. By the widely used method which is based on the normal form theory and the center manifold theorem introduced by Hassard et al. [9].The direction, stability, and the period of the bifurcating periodic solutions can be obtain. On the other hand, from [13,26,27], we known that the properties of Hopf bifurcation of discrete schemes are the same as that of the corresponding delay differential equations, such as Runge-Kutta or strictly stable linear multistep method. In the last section, we will use some numerical simulations to illustrate the analytical results we obtained in previous sections and to show the properties of Hopf bifurcation, such as the direction of bifurcation and stability of periodic solutions. 


\section{The existence and normal form of the double Hopf bi- furcation}

In this section, for system (3.1), the existence of the double Hopf bifurcation from origin $(0,0)$ is obtained and the normal form will be also described. In the above section, we find that the system (3.1) undergoes a Hopf bifurcation at the origin $(0,0)$ when $\sigma=\sigma_{j}^{ \pm}, j=0,1, \ldots$ Thus, a possible double Hopf bifurcation occurs when some $\sigma_{0}=\sigma_{j}^{+}=\sigma_{l}^{-}$, where $j, l=0,1, \ldots$. The equality $\sigma=\sigma_{j}^{+}=\sigma_{l}^{-}$implies that the linearized system on the trivial equilibrium has two pairs of purely imaginary eigenvalues $\pm i \omega_{-} \sigma_{0}$ and $\pm i \omega_{+} \sigma_{0}$. In this paper, we assume $\omega_{-}: \omega_{+} \neq n_{1}: n_{2} \in Z_{+}$. That is, we only consider the non-resonant double Hopf bifurcation.

We consider the value $a$ and time delay $\sigma$ as bifurcation parameters of system (3.1). Suppose system (3.1) undergoes a double Hopf bifurcation from the trivial equilibrium at the critical point: $a=a_{0}, \sigma=\sigma_{0}$. From Theorem 4, If $H>0, \Delta>0$ and $\left(H_{1}\right)$ is satisfied. Then the equation (3.9) has two roots with positive and negative real parts when $\sigma=0$, respectively. Therefore, we have reason to believe the existence of such possible situation: its roots except $\pm i \omega_{-} \sigma_{0}$ and $\pm i \omega_{+} \sigma_{0}$ have negative real parts at the first intersection point by the transverse condition. Thus, the center manifold can be used to describe the dynamics of the whole system in this case.

Rescaling the time by $t \mapsto \frac{t}{\sigma}$ to normalize the delay, and set $a=a_{0}+\alpha_{1}$ and $\sigma=\sigma_{0}+\alpha_{2}$.

The system (3.1) may be transformed into a functional differential equation (FDE) in $C=C\left([-1,0], R^{2}\right)$, where the phase space $C=C\left([-1,0], R^{2}\right)$ as the banach space of continuous functions from $[-1,0]$ to $R^{2}$ with the supremum norm (see [8]) as

$$
\frac{d U}{d t}=L_{\mu}\left(U_{t}\right)+F\left(\mu, U_{t}\right)
$$

where $U(t)=\left(u_{1}(t), u_{2}(t)\right)^{T} \in R^{2}, U_{t} \in C$ is defined by

$$
U_{t}=U(t+\theta),-1 \leq \theta \leq 0, \quad L_{\mu}: C \rightarrow R^{2}, F: R \times C \rightarrow R^{2}, \alpha=\left(\alpha_{1}, \alpha_{2}\right)^{T} .
$$

When $\alpha=(0,0)^{T}$, then we have $L_{0}(\phi)$ and $F(0, \phi)$ respectively by

$$
L_{0}(\phi)=B_{0} \phi(0)+B_{1} \phi(-1) .
$$

We set

$$
B_{0}=\sigma_{0}\left(\begin{array}{cc}
0 & \frac{b}{k_{A}} \\
k_{A} & -k_{d A}
\end{array}\right), B_{1}=\sigma_{0}\left(\begin{array}{cc}
-a_{0} & 0 \\
0 & 0
\end{array}\right)
$$

and

$$
F(0, \phi)=\sigma_{0}\left(\begin{array}{c}
-v_{1} \phi_{1}(0) \phi_{1}(-1)+v_{2} \phi_{2}^{2}(0)-v_{3} \phi_{2}^{3}(0)+h . o . t . \\
0
\end{array}\right),
$$

where $v_{1}=\frac{\mu}{K}, v_{2}=\frac{\gamma k_{d A} A^{*}}{k_{A}\left(\beta+A^{*}\right)^{3}}, v_{3}=\frac{\gamma k_{d A} A^{*}}{k_{A}\left(\beta+A^{*}\right)^{4}}$. Then when $\alpha=(0,0)^{T}$, the linearization equation at the trivial equilibrium of (3.1) is

$$
\frac{d U}{d t}=L_{0}\left(U_{t}\right)
$$


and the bilinear form on $C^{*} \times C$ is

$$
(\psi(s), \phi(\theta))=\psi(0) \phi(0)-\int_{-1}^{0} \int_{0}^{\theta} \psi(\xi-\theta) d \eta(\theta) \phi(\xi) d \xi
$$

where

$$
\eta(\theta)=\left\{\begin{array}{l}
B_{0}, \theta=0, \\
-B_{1}, \theta=-1, \quad \phi(\theta)=\left(\phi_{1}(\theta), \phi_{2}(\theta)\right) \in C, \psi(s)=\left(\psi_{1}(s), \psi_{2}(s)\right)^{T} \in C^{*} . \\
0, \theta \in(-1,0),
\end{array}\right.
$$

Then, the phase space $C$ is decomposed by $\pm i \omega_{-} \sigma_{0}, \pm i \omega_{+} \sigma_{0}$ as $C=P \bigoplus Q$, where $P$ is the center subspace spanned by the basis vectors associated with the imaginary characteristic roots and $Q$ is the complement subspace of $P$.

We now consider the enlarged phase space $\mathrm{BC}$ of functions from $[-1,0]$ to $R^{2}$, which are continuous on $[-1,0)$ with a possible jump discontinuity at zero. This space can be identified as $C \times R^{2}$. Elements of it can be written in the form $\phi+X_{0} \eta$, where $\phi \in C, \eta \in R^{2}$ and $X_{0}(\theta)=0$ for $\theta \in[-1,0)$ and $X_{0}(0)=I$. Define a continuous projection $\pi: B C \rightarrow P$ as $\pi\left(\phi+X_{0} \eta\right)=\Phi[(\Psi, \Phi)+\Psi(0) \eta]$. Then, the the bases of $P$ and $P^{*}$ can be computed directly that

$$
\begin{aligned}
& \Phi(\theta)=\left(\begin{array}{cccc}
e^{i \omega_{-} \sigma_{0} \theta} & e^{-i \omega_{-} \sigma_{0} \theta} & e^{i \omega_{+} \sigma_{0} \theta} & e^{i \omega_{+} \sigma_{0} \theta} \\
A_{1} & A_{2} & A_{3} & A_{4}
\end{array}\right),
\end{aligned}
$$

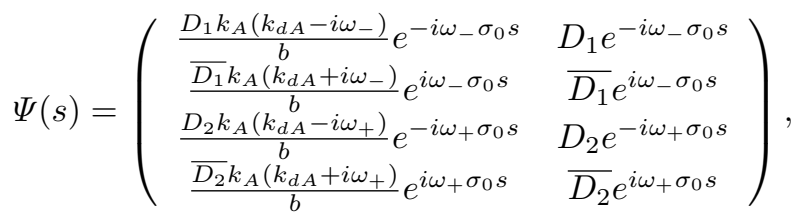

where

$$
\begin{aligned}
& A_{1}=\frac{a_{0} k_{A} e^{-i \omega_{-} \sigma_{0}}+i \omega_{-} k_{A}}{b} e^{i \omega_{-} \sigma_{0} \theta}, \quad A_{2}=\frac{a k_{A} e^{i \omega_{-} \sigma_{0}}-i \omega_{-} k_{A}}{b} e^{-i \omega_{-} \sigma_{0} \theta}, \\
& A_{3}=\frac{a k_{A} e^{i \omega_{+} \sigma_{0}}+i \omega_{+} k_{A}}{b} e^{i \omega_{+} \sigma_{0} \theta}, \quad A_{4}=\frac{a_{0} k_{A} e^{i \omega_{+} \sigma_{0}}-i \omega_{+} k_{A}}{b} e^{-i \omega_{+} \sigma_{0} \theta} \\
& D_{1}=\frac{b}{k_{A}\left(k_{d A}+i \omega_{-} a \sigma_{0}-a k_{d A} \sigma_{0}+a_{0} e^{\left.-i \omega_{-} \sigma_{0}\right)}\right.}, \\
& D_{2}=\frac{b}{k_{A}\left(k_{d A}+i \omega_{+} a_{0} \sigma_{0}-a k_{d A} \sigma_{0}+a_{0} e^{\left.-i \omega_{+} \sigma_{0}\right)}\right.}
\end{aligned}
$$

Thus from the define of the bilinear inner product, we have $(\Psi(0), \Phi(0))=I$. Note that the parameter $\alpha=\left(\alpha_{1}, \alpha_{2}\right)$, when it has a small perturbations at zero, the system (3.1) can be written as

$$
\dot{U(t)}=L_{\alpha} U_{t}+F\left(U_{t}, \alpha\right)
$$

where

$$
\begin{aligned}
L_{\alpha}\left(U_{t}\right)=\left(\sigma_{0}+\alpha_{2}\right)\left(\begin{array}{cc}
0 & \frac{b}{k_{A}} \\
k_{A} & -k_{d A}
\end{array}\right)\left(\begin{array}{l}
u_{1 t}(0) \\
u_{2 t}(0)
\end{array}\right) \\
+\left(\sigma_{0}+\alpha_{2}\right)\left(a_{0}+\alpha_{1}\right)\left(\begin{array}{cc}
-1 & 0 \\
0 & 0
\end{array}\right)\left(\begin{array}{l}
u_{1 t}(-1) \\
u_{2 t}(-1)
\end{array}\right)
\end{aligned}
$$


and

$$
F\left(U_{t}, \alpha\right)=\left(\sigma_{0}+\alpha_{2}\right)\left(\begin{array}{c}
-v_{1} u_{1}(0) u_{1}(-1)+v_{2} u_{2}^{2}(0)-v_{3} u_{2}^{3}(0)+\text { h.o.t. } \\
0
\end{array}\right) .
$$

By the continuous projection $\pi$, we can decompose the enlarged phase space by $\pm i \omega_{-}, \pm i \omega_{+}$as $B C=P \oplus K e r \pi$. Let $U_{t}=\Phi z(t)+v(t)$, then (4.1) is therefore decomposed as the system

$$
\begin{aligned}
\dot{z(t)} & =B z+\Psi(0) \widetilde{F}(\Phi z+v, \alpha) \\
\dot{v} & =A_{Q^{1}} v+(I-\pi) X_{0} \widetilde{F}(\Phi z+v, \alpha),
\end{aligned}
$$

where $B=\operatorname{diag}\left\{i \omega_{-} \sigma_{0},-i \omega_{-} \sigma_{0}, i \omega_{+} \sigma_{0},-i \omega_{+} \sigma_{0}\right\}, A_{Q^{1}}$ is the restriction of $A$ as an operator from $Q^{1}:=Q \bigcap C^{1}$ to the Banach space $\operatorname{Ker} \pi$ with $A: C^{1} \rightarrow$ $B C, A U=\dot{U}+X_{0}\left[L_{0} U-\dot{U}(0)\right]$ and $\widetilde{F}(U, \alpha)=\left[L_{\alpha}-L_{0}\right] U+F(U, \alpha), v \in Q^{1}$. Neglecting higher order terms with respect to parameters $\alpha$, the above can be written as

$$
\left\{\begin{array}{l}
\dot{z}_{1}=i \sigma_{0} \omega_{-} z_{1}+\sum_{j=1}^{2} \psi_{1 j}\left(F_{2}^{j}+F_{3}^{j}\right)+\text { h.o.t., } \\
\dot{z_{2}}=-i \sigma_{0} \omega_{-} z_{2}+\sum_{j=1}^{2} \psi_{2 j}\left(F_{2}^{j}+F_{3}^{j}\right)+\text { h.o.t. } \\
\dot{z_{3}}=i \sigma_{0} \omega_{+} z_{3}+\sum_{j=1}^{2} \psi_{3 j}\left(F_{2}^{j}+F_{3}^{j}\right)+\text { h.o.t., } \\
\dot{z_{4}}=i \sigma_{0} \omega_{+} z_{4}+\sum_{j=1}^{2} \psi_{4 j}\left(F_{2}^{j}+F_{3}^{j}\right)+\text { h.o.t. } \\
\dot{v}=A_{Q^{1}} v+(I-\pi) X_{0} \widetilde{F}(\Phi z+v, \alpha),
\end{array}\right.
$$

where

$$
\begin{aligned}
F_{2}^{1}= & \frac{\alpha_{2}}{k_{A}}\left[\left(a_{0} k_{A} e^{-i \omega_{-} \sigma_{0}}+i \omega_{-}\right) z_{1}+\left(a_{0} k_{A} e^{i \omega_{-} \sigma_{0}}-i \omega_{-}\right) z_{2}\right. \\
& \left.+\left(a_{0} k_{A} e^{-i \omega_{+} \sigma_{0}}+i \omega_{+}\right) z_{3}+\left(a_{0} k_{A} e^{i \omega_{+} \sigma_{0}}-i \omega_{+}\right) z_{4}+b v_{2}(0)\right] \\
& -\left(a_{0} \alpha_{2}+\sigma_{0} \alpha_{1}\right)\left[z_{1} e^{-i \omega_{-} \sigma_{0}}+z_{2} e^{i \omega_{-} \sigma_{0}}+z_{3} e^{-i \omega_{+} \sigma_{0}}+z_{4} e^{i \omega_{+} \sigma_{0}}+v_{1}(-1)\right], \\
F_{2}^{2}= & \alpha_{2} k_{A}\left[z_{1}+z_{2}+z_{3}+z_{4}+v_{2}(0)\right]-\frac{\alpha_{2} k_{d A}}{b}\left[\left(a_{0} k_{A} e^{-i \omega_{-} \sigma_{0}}+i \omega_{-}\right) z_{1}\right. \\
& +\left(a_{0} k_{A} e^{i \omega_{-} \sigma_{0}}-i \omega_{-}\right) z_{2}+\left(a_{0} k_{A} e^{-i \omega_{+} \sigma_{0}}+i \omega_{+}\right) z_{3} \\
& \left.+\left(a_{0} k_{A} e^{i \omega_{+} \sigma_{0}}-i \omega_{+}\right) z_{4}+b v_{2}(0)\right] \\
F_{3}^{1}= & \left(\sigma_{0}+\alpha_{2}\right)\left[-v_{1}\left(z_{1}+z_{2}+z_{3}+z_{4}+v_{1}(0)\right)\left(z_{1} e^{-i \omega_{-} \sigma_{0}}+z_{2} e^{i \omega_{-} \sigma_{0}}\right.\right. \\
& \left.+z_{3} e^{-i \omega_{+} \sigma_{0}}+z_{4} e^{i \omega_{+} \sigma_{0}}+v_{1}(-1)\right)+v_{2}\left(z_{1}+z_{2}+z_{3}+z_{4}+v_{1}(0)\right)^{2} \\
& \left.-v_{3}\left(z_{1}+z_{2}+z_{3}+z_{4}+v_{1}(0)\right)^{3}+O\left(\|u\|^{4}\right)\right] \\
F_{3}^{2}= & 0 .
\end{aligned}
$$


Next, let $M_{2}^{1}$ denote the operator defined in $V_{2}^{6}\left(C^{4} \times K e r \pi\right)$, with $M_{2}^{1}$ : $V_{2}^{6}\left(C^{4}\right) \mapsto V_{2}^{6}\left(C^{4}\right)$ where $V_{2}^{6}\left(C^{4}\right.$ represents the linear space of the second order homogeneous polynomials in six variables $\left(z_{1}, z_{2}, z_{3}, z_{4}, \alpha_{1}, \alpha_{2}\right)$ with coefficients in $C^{4}$. Then, it is easy to verify that one may choose the decomposition $V_{2}^{6}\left(C^{4}\right)=\operatorname{Im}\left(M_{2}^{1}\right) \bigoplus \operatorname{Im}\left(M_{2}^{1}\right)^{c}$ with complementary space $\operatorname{Im}\left(M_{2}^{1}\right)^{c}$ spanned by the elements $z_{j} \alpha_{i} e_{j}, i=1,2, j=1,2,3,4$, where $e_{j}(j=1,2,3,4)$ are unit vectors.

Then the normal form of equation (4.1) on the center manifold of the origin near $\alpha=0$ up to quadratic order terms is given by

$$
\dot{z}=B z+\frac{1}{2} g_{2}^{1}(z, 0, \alpha)
$$

where $g_{2}^{1}(z, 0, \alpha)$ is determined by $g_{2}^{1}(z, 0, \alpha)=\operatorname{Proj}_{\left(\operatorname{Im}\left(M_{2}^{1}\right)\right)^{c}} \times f_{2}^{1}(z, 0, \alpha)$ with $f_{2}^{1}(z, 0, \alpha)$ is the function giving the quadratic terms in $(z, \alpha)$ for $v=0$ defined by the first equation of (4.2). Thus, Neglecting the conjugate situation, we only consider $z_{1}$ and $z_{3}$, the normal form (4.4) becomes that

$$
\left\{\begin{array}{l}
\dot{z}_{1}=i \sigma_{0} \omega_{-} z_{1}+b_{1} z_{1} \\
\dot{z_{3}}=i \sigma_{0} \omega_{+} z_{3}+b_{3} z_{3}
\end{array},\right.
$$

where

$$
\begin{aligned}
b_{1}= & \frac{D_{1}}{b}\left(k_{A} \alpha_{2} b-\mathrm{e}^{-i \sigma_{0} \omega_{-}} k_{d A} a_{0} k_{A} \alpha_{2}+\alpha_{2} \omega_{-}{ }^{2}\right. \\
& \left.-\mathrm{e}^{-i \sigma_{0} \omega_{-}} \sigma_{0} \alpha_{1} k_{A} k_{d A}+i \mathrm{e}^{-i \sigma_{0} \omega_{-}} \sigma_{0} \alpha_{1} k_{A} \omega_{-}\right), \\
b_{3}= & \frac{D_{2}}{b}\left(k_{A} \alpha_{2} b-\mathrm{e}^{-i \sigma_{0} \omega_{+}} k_{d A} a_{0} k_{A} \alpha_{2}+\alpha_{2} \omega_{+}{ }^{2}\right. \\
& \left.-\mathrm{e}^{-i \sigma_{0} \omega_{+}} \sigma_{0} \alpha_{1} k_{A} k_{d A}+i \mathrm{e}^{-i \sigma_{0} \omega_{+}} \sigma_{0} \alpha_{1} k_{A} \omega_{+}\right) .
\end{aligned}
$$

Next, we need calculate higher order terms of the normal form. Similarly, let $M_{3}^{1}$ denote the operator defined in $V_{3}^{4}\left(C^{4} \times K e r \pi\right)$ with

$$
M_{3}^{1}: V_{3}^{4} \mapsto V_{3}^{4},\left(M_{3}^{1}\right) p(z, \alpha)=D_{z} p(z, \alpha) B z-B p(z, \alpha),
$$

where $V_{3}^{4}$ denotes the linear space of the third order homogeneous polynomials in four variables $\left(z_{1}, z_{2}, z_{3}, z_{4}\right)$. When there is no strong resonance, it is easy to check that one may choose the decomposition $V_{3}^{4}=\left(\operatorname{Im} M_{3}^{1}\right) \bigoplus\left(\operatorname{Im} M_{3}^{1}\right)^{c}$ with complementary space $\left(\operatorname{Im} M_{3}^{1}\right)^{c}$ spanned by the elements

$$
z_{1}^{2} z_{2} e_{1}, z_{1} z_{3} z_{4} e_{1}, z_{1} z_{2}^{2} e_{2}, z_{2} z_{3} z_{4} e_{2}, z_{1} z_{2} z_{3} e_{3}, z_{3}^{2} z_{4} e_{3}, z_{1} z_{2} z_{4} e_{4}, z_{3} z_{4}^{2} e_{4} .
$$

Then we can derive the normal form up to the third order

$$
\dot{z}=B z+\frac{1}{2} g_{2}^{1}(z, 0, \alpha)+\frac{1}{6} g_{3}^{1}(z, 0, \alpha),
$$

where

$$
g_{3}^{1}(z, 0,0)=\left(I-P_{I, 3}^{1}\right) f_{3}^{1}(z, 0,0)
$$


and $f_{3}^{1}(z, 0,0)$ is the function giving the cubic terms in $(z, \alpha)$ for $v=0, \alpha=0$ defined by the first equation of (4.2). Then, equation (4.5) can be written as

$$
\left\{\begin{array}{l}
\dot{z_{1}}=i \sigma_{0} \omega_{-} z_{1}+b_{1} z_{1}+b_{112} z_{1}\left|z_{1}\right|^{2}+b_{134} z_{1}\left|z_{3}\right|^{2}, \\
\dot{z_{3}}=i \sigma_{0} \omega_{+} z_{3}+b_{3} z_{3}+b_{334} z_{3}\left|z_{3}\right|^{2}+b_{123} z_{3}\left|z_{1}\right|^{2},
\end{array}\right.
$$

where

$$
\begin{array}{ll}
b_{112}=-\frac{3 D_{1} k_{A} \sigma_{0} v_{3}\left(k_{d A}-i \omega_{-}\right)}{b}, & b_{134}=-\frac{6 D_{1} k_{A} \sigma_{0} v_{3}\left(k_{d A}-i \omega_{-}\right)}{b}, \\
b_{334}=-\frac{3 D_{2} k_{A} \sigma_{0} v_{3}\left(k_{d A}-i \omega_{+}\right)}{b}, & b_{123}=-\frac{6 D_{2} k_{A} \sigma_{0} v_{3}\left(k_{d A}-i \omega_{+}\right)}{b} .
\end{array}
$$

In polar coordinates $z_{1}=r_{1} e^{i \theta_{1}}, z_{3}=r_{2} e^{i \theta_{2}}$, the amplitude equation resulted from equation (4.6) is

$$
\left\{\begin{array}{l}
\dot{r_{1}}=\operatorname{Re}\left(b_{1}\right) r_{1}+\operatorname{Re}\left(b_{112}\right) r_{1}^{3}+\operatorname{Re}\left(b_{134}\right) r_{1} r_{2}^{2}, \\
\dot{r_{2}}=\operatorname{Re}\left(b_{3}\right) r_{2}+\operatorname{Re}\left(b_{334}\right) r_{2}^{3}+\operatorname{Re}\left(b_{123}\right) r_{2} r_{1}^{2} .
\end{array}\right.
$$

\section{$5 \quad$ Numerical Simulations}

In this section, we use some numerical simulations to illustrate the analytical results we obtained in previous sections.

We first consider system (1.1) with $\mu=0.4, K=0.3, \gamma=0.3, k_{d A}=$ $0.09, \beta=0.6$, and $k_{A}=0.5, \sigma=0$. Under these parameter values, system undergoes a Hopf bifurcation when $\tau \doteq 8.93635$. By Theorem 3, the equilibrium $E^{*}$ is unstable for all $\tau \geq 0$.

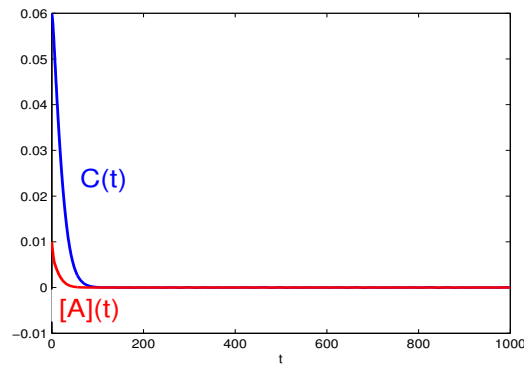

Figure 1. Simulation solution of (1.1):the equilibrium $E_{0}$ is asymptotically stable, when $\tau=4.5<\tau_{0}$.

As shown in Figure 1, when $\tau<\tau_{0}$, the equilibrium $E^{*}$ is unstable and the other equilibrium $E_{0}$ is asymptotically stable. That is, when the killing module and rescue module are both on, for small specific growth rate $\mu<\frac{\gamma}{\beta}$, the the bacterial will become extinct at last. We choose we choose $\tau=9.5>\tau_{0}$, Figure 2 shows that there a periodic solution with small amplitude exists. A strong Allee effect is observed, the specific growth rate is negative if the initial 
$C$ is below the Allee threshold (see Figure 1). When the initial $C$ is above the Allee threshold, although the time of rescue delay is large, the density of E. Coli. has oscillation near the threshold.

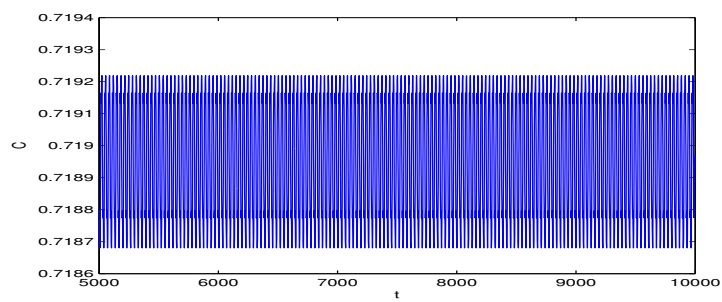

(a)

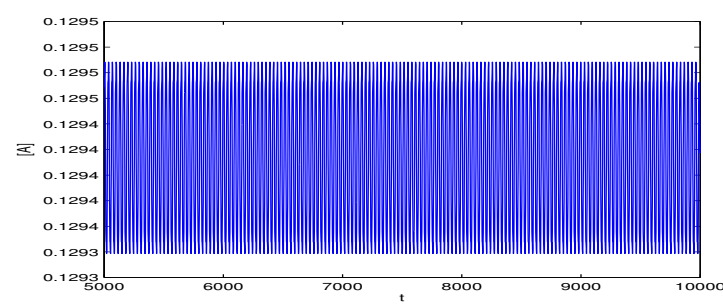

(b)

Figure 2. Simulation solution of (1.1): a periodic solution is bifurcated, when $\tau=9.5>\tau_{0}$.

Next, we change the values of $\gamma$ to 0.05 , i.e., the killing rate of the circuit is reduced. Hence, it follows that $H<0$ and $\mu>\frac{\gamma}{\beta}$. Now, we know that $E_{0}$ becomes a saddle from sink and $E^{*}$ is a sink. Figure 3 shows that the stability of $E^{*}$.

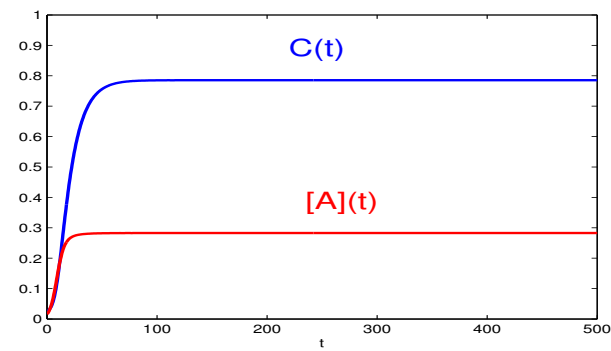

Figure 3. Simulation solution of (1.1):the equilibrium $E^{*}$ is asymptotically stable, when $\tau=5$.

At last, we can give some analyses and examples to observe the behavior near the double Hopf bifurcation of system (4.1). For convenience, we assume that $a$ is a independent parameter. Choosing $\mu=0.4, K=0.3, \gamma=$ 
$0.3, k_{d A}=0.01, \beta=0.6, k_{A}=0.5, \tau=0$, then $b=0.0008147257349$. We can obtain $\sigma_{0}=24.8210724, a_{0}=0.0714267$. The unfolding parameters are $\operatorname{Re}\left(b_{1}\right)=1.4246752743 \alpha_{1}+0.3245334987 \alpha_{2}$ and $\operatorname{Re}\left(b_{3}\right)=2.2157433961 \alpha_{1}-$ $0.1126549270 \alpha_{2}$. we also obtain that $\operatorname{Re}\left(b_{112}\right)=6.4350970712, \operatorname{Re}\left(b_{134}\right)=$ $12.87019414, \operatorname{Re}\left(b_{123}\right)=-7.300691906, \operatorname{Re}\left(b_{334}\right)=-3.6503459527$. Thus, the pdifficultq case happens under the parameters above. Let $\rho_{1}=\operatorname{Re}\left(b_{112}\right) r_{1}^{2}, \rho_{2}=$ $-\operatorname{Re}\left(b_{334}\right) r_{2}^{2}, \tilde{t}=2 t$, then we have the following planar system in terms of $\rho_{1}$ and $\rho_{2}$ :

$$
\left\{\begin{array}{l}
\dot{\rho_{1}}=\rho_{1}\left(\operatorname{Re}\left(b_{1}\right)+\rho_{1}-\frac{\operatorname{Re}\left(b_{134}\right)}{\operatorname{Re}\left(b_{334}\right)} \rho_{2}\right), \\
\dot{\rho_{2}}=\rho_{2}\left(\operatorname{Re}\left(b_{3}\right)-\rho_{2}+\frac{\operatorname{Re}\left(b_{123}\right)}{\operatorname{Re}\left(b_{112}\right)} \rho_{1}\right) .
\end{array}\right.
$$

Note that $N_{0}=(0,0)$ is always an equilibrium of (5.1). The other two semi-trivial equilibria given in terms of perturbation parameters are $N_{1}=$ $\left(-\operatorname{Re}\left(b_{1}\right), 0\right)$ and $N_{2}=\left(0, \operatorname{Re}\left(b_{3}\right)\right)$. There also exist a nontrivial equilibrium $N_{3}=\left(\frac{\operatorname{Re}\left(b_{1}\right)+3.52574641}{3}, \frac{\operatorname{Re}\left(b_{3}\right)+1.13451154}{-3}\right)$. Since there does not exist unstable manifold containing the equilibrium, according to the center manifold theory, the solutions on the center manifold determine the asymptotic behavior of the solutions of the system (4.1). Therefore, if equation (5.1) has stable(unstable) semi-trivial equilibria, then (4.1) has stable(unstable) periodic solutions in the neighborhood of the trivial equilibrium. Since equation (5.1) has a nontrivial equilibrium $N_{3}$, then (4.1) has a quasi-periodic solution in the neighborhood of $(0,0)$. So, we shall call the periodic solution or the quasi-periodic solution the source (respectively, saddle, sink) periodic solution of (4.1) if the semi-trivial equilibrium or the nontrivial equilibrium of (5.1) is a source (respectively, saddle, sink), respectively. The corresponding bifurcation diagram and phase portraits are shown in the parameter plane $\left(\alpha_{1}, \alpha_{2}\right)$ by Figure 4, where the critical bifurcation lines $L_{1}: \alpha_{2}=-4.389917466 \alpha_{1}, L_{2}: \alpha_{2}=19.66841092 \alpha_{1}, L_{3}$ : $\alpha_{2}=127.1253084 \alpha_{1}, L_{4}: \alpha_{2}=-14.99637164 \alpha_{1}, L_{5}: \alpha_{2}=-71.46428697 \alpha_{1}$. We explain the bifurcations in the anticlockwise direction, starting from region (1). First, in region (1), there is only one trivial equilibrium which is a saddle. When the parameters are varied across the line $L_{1}$ from region (1) to (2), $N_{0}$ becomes a sink, and anther semi-trivial equilibria $N_{1}$ (saddle) appears. That is, for the system (4.1), an periodic solution(saddle) appears due to Hopf bifurcation. In the region (3), a more sink $N_{2}$ exists, i.e., another periodic solution(sink) appears, and $N_{0}$ becomes a saddle. When the parameters are varied from region (3) to region (4), $N_{2}$ becomes a saddle, a new focus $N_{3}$ appears which corresponding a quasi-periodic solution of the system (4.1). When the parameters are varied from region (4) to region (5), a limit cycle is present in the system (5.1). At this time, for system (4.1), a torus may be appears. When the parameters are further changed from region (5) to region (6), the limit cycle disappears, $N_{3}$ collides with $N_{1}$ and then also disappears, and $N_{1}$ becomes a source. From region (6) to region (7), $N_{1}$ disappears, and the $N_{0}$ becomes a source. When the parameters to region (1), $N_{2}$ disappears at last. Moreover, a heteroclinic cycle may be formed between $N_{1}$ and $N_{2}$, which is the main reason of the disappears of limit cycle. 


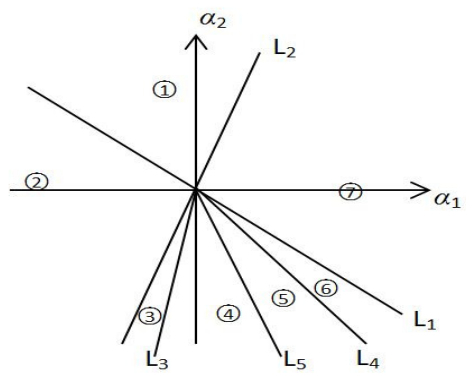

(a)
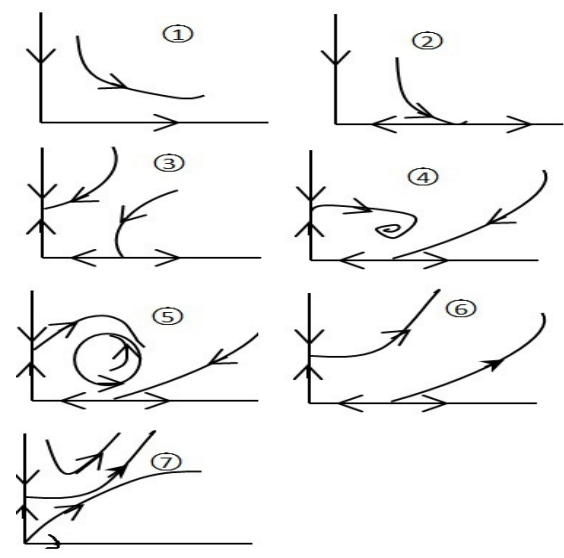

(b)

Figure 4. The bifurcation diagram and phase portraits for (5.1) near $\left(\alpha_{1}, \alpha_{2}\right)=(0,0)$.

To demonstrate the analytic results obtained in above, here we present some interest numerical simulation results. We choose there groups of perturbation parameter values: $\left(\alpha_{1}, \alpha_{2}\right)=(-0.001 .0 .021),(0.001,-0.075),(0.001,-0.050)$, belonging to the regions (3),(4) and (5), corresponding to a stable periodic solution as depicted in Figure 5, a stable quasi-periodic solution, see Figure 6, and a torus displayed in the three dimensional $u_{1}-u_{2}-u_{1}^{\prime}$ space, see Figure 7. It is clear that the numerical simulations agree very well with the analytical predictions.

\section{Conclusions}

In this paper, we have discussed the dynamical behaviors of a delay differential model which confers a strong Allee effect in Escherichia coli. Firstly, we have shown that the Hopf bifurcation exists as the time delay $\tau$ or $\sigma$ crosses some critical values.

Secondly, we have considered the double Hopf bifurcation in system (4.1) 


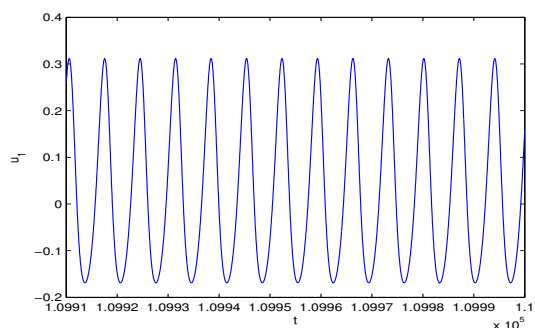

(a)

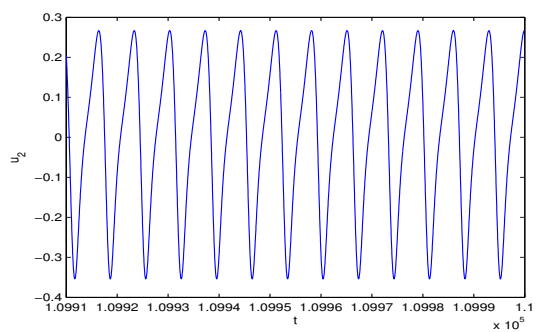

(b)

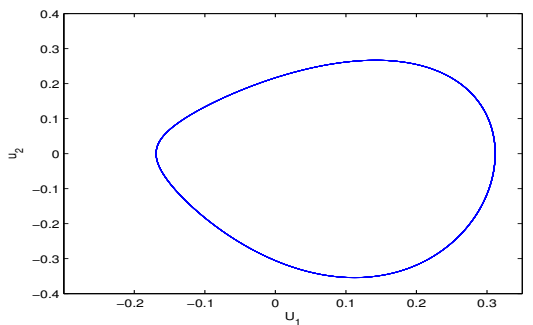

(c)

Figure 5. Simulated solution of system $(4.1)$ for $\left(\alpha_{1}, \alpha_{2}\right)=(-0.001 .-0.075)$ : showing a stable periodic solution.

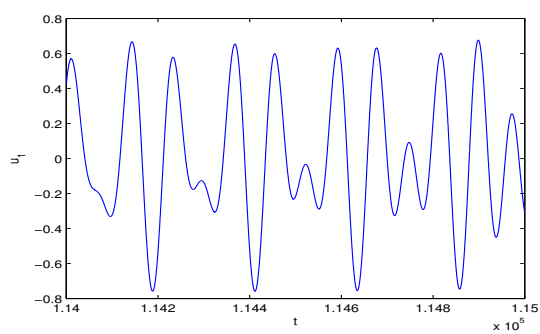

(a)

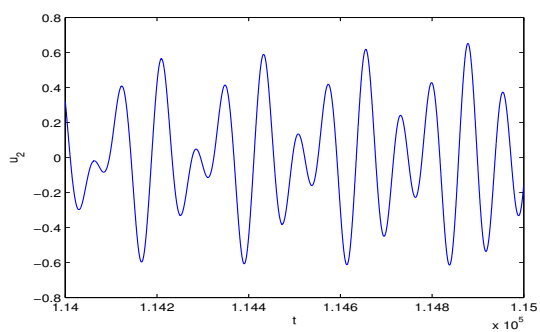

(b)

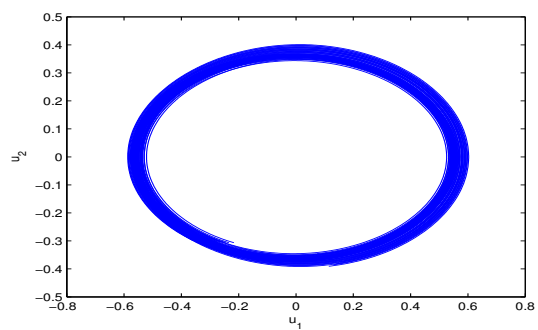

(c)

Figure 6. Simulated solution of system (4.1) for $\left(\alpha_{1}, \alpha_{2}\right)=(-0.001 .0 .021)$ : showing a stable quasi-periodic solution. 


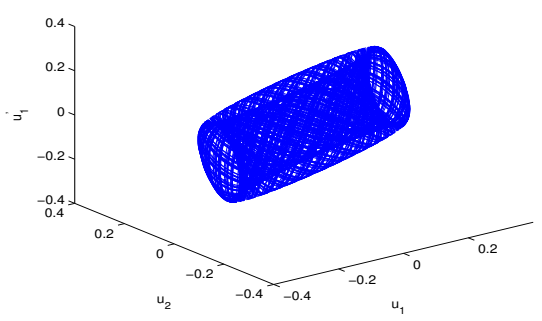

Figure 7. Simulation solution of $(4.1)$ for $\left(\alpha_{1}, \alpha_{2}\right)=(0.001 .-0.050)$ : showing a torus in $u_{1}-u_{2}-u_{1}^{\prime}$ space.

with delay $\sigma$. We have obtained the vector field reduced to the center manifold, and derived the normal forms and their unfolding with perturbation parameters. Furthermore, we have given the bifurcation diagram as the unfolding parameter values are near the critical point.

We improved the model in [20], and the research presented is a new step about our system in studied from the view point of high co-dimensional bifurcations. The study will help understanding the interpreting biological phenomena in theory.

\section{Acknowledgements}

The research was supported by the National Natural Sciences Foundation of PR China (no.11372196) and the Youth Foundation of Hebei Educational of Committee Grant (no. QN2014041). The authors wish to express their thanks to the anonymous referee for many valuable remarks which improve the manuscript greatly.

\section{References}

[1] W.C. Allee. Animal aggregations, a study in general sociology. Univ. of Chicago Press, Chicago, 1931. http://dx.doi.org/10.5962/bhl.title.7313.

[2] E. Beretta and Y. Kuang. Geometric stability switch criteria in delay differential systems with delay dependent parameters. SIAM Journal on Mathematical Analysis, 33(5):1144-1165, 2002. http://dx.doi.org/10.1137/S0036141000376086.

[3] D. S. Boukal and L. Berec. Single-species models of the Allee effect: extinction boundaries, sex ratios and mate encounters. Journal of Theoretical Biology, 218(3):375-394, 2002. http://dx.doi.org/10.1006/jtbi.2002.3084.

[4] R. Čiegis and A. Bugajev. Numerical approximation of one model of bacterial self-organization. Nonlinear Analysis: Modelling and Control, 17(3):253-270, 2012 .

[5] F. Courchamp, T. Clutton-Brock and Bryan Grenfell. Inverse density dependence and the Allee effect. Trends in Ecology and Evolution, 14(10):405-410, 1999. http://dx.doi.org/10.1016/S0169-5347(99)01683-3. 
[6] T. Faria and L.T. Magalhães. Normal forms for retarded functional differential equations and applications to Bogdanov-Takens singularity. Journal of Differential Equations, 122(2):201-224, 1995. http://dx.doi.org/10.1006/jdeq.1995.1145.

[7] T. Faria and L.T. Magalhães. Normal forms for retarded functional differential equations with parameters and applications to Hopf bifurcation. Jour. of Diff. Eq., 122(2):181-200, 1995. http://dx.doi.org/10.1006/jdeq.1995.1144.

[8] J.K. Hale and S. M. Verduyn Lunel. Introduction to functional differential equations. Springer, New York, 1993. http://dx.doi.org/10.1007/978-1-4612-4342-7.

[9] B.D. Hassard, N.D. Kazarinoff and Y.H. Wan. Theory and Applications of Hopf Bifurcation. Cambridge Univ. Press, Cambridge, UK, 1981.

[10] E.E. Holmes, M. A. Lewis, J. E. Banks and R. R. Veit. Partial differential equations in ecology: spatial interactions and population dynamics. Ecology, 75(1):17-29, 1994. http://dx.doi.org/10.2307/1939378.

[11] N. Hritonenko and Y. Yatsenko. Mathematical Modeling in economics, ecology and the environment. Springer, New York, 2013. http://dx.doi.org/10.1007/9781-4614-9311-2.

[12] Y. Kuang. Delay differential equations: with applications in population dynamics, volume 191. Academic Press, New York, 1993.

[13] M. Z. Liu and Q. Wang. Numerical Hopf bifurcation of linear multistep methods for a class of delay differential equations. Applied Mathematics and Computation, 208(2):462-474, 2009. http://dx.doi.org/10.1016/j.amc.2008.12.013.

[14] M. B. Miller and B. L. Bassler. Quorum sensing in bacteria. Annual Reviews in Microbiology, 55:165-199, 2001. http://dx.doi.org/10.1146/annurev.micro.55.1.165.

[15] R. A. Myers, N. J. Barrowman, J. A. Hutchings and A. A. Rosenberg. Population dynamics of exploited fish stocks at low population levels. Science, 269:11061108, 1995.

[16] H. T. Odum and W. C. Allee. A note on the stable point of populations showing both intraspecific cooperation and disoperation. Ecology, 35(1):95-97, 1954. http://dx.doi.org/10.2307/1931412.

[17] K. J. Painter and T. Hillen. Spatio-temporal chaos in a chemotaxis model. Phys. D: Non. Phen., 240(4-5):363-375, 2011. http://dx.doi.org/10.1016/j.physd.2010.09.011.

[18] L. Roques, J. Garnier, F. Hamel and E. K. Klein. Allee effect promotes diversity in traveling waves of colonization. Proceedings of the National Academy of Sciences of USA, 109(23):8828-8833, 2012. http://dx.doi.org/10.1073/pnas.1201695109.

[19] S. Ruan. Delay Differential Equations and Applications in Single Species Dynamics. Springer, Berlin, 2006.

[20] R. Smith, Ch. Tan, J.K. Srimani, A. Pai, K. A. Riccione, H. Song and L. You. Programmed Allee effect in bacteria causes a tradeoff between population spread and survival. Proceedings of the National Academy of Sciences of USA, 111(5):1969-1974, 2014. http://dx.doi.org/10.1073/pnas.1315954111.

[21] M. E. Soulé and B. A. Wilcox. Conservation biology: an evolutionary-ecological perspective. Sinauer Associates, Sunderland Massachusetts, 1980. 
[22] P. A. Stephens, W. J. Sutherland and R. P. Freckleton. What is the Allee effect? Oikos, 87(1):185-190, 1995.

[23] Y. Su, J. Wei and J. Shi. Hopf bifurcations in a reaction-diffusion population model with delay effect. Journal of Differential Equations, 247(4):1156-1184, 2009. http://dx.doi.org/10.1016/j.jde.2009.04.017.

[24] Q. Wang. Stability analysis in a second-order differential equation with delays. International Journal of Computer Mathematics, 89(10):1345-1354, 2012. http://dx.doi.org/10.1080/00207160.2012.680447.

[25] Q. Wang. Stability and bifurcation of the Tsodyks-Markram model about shortterm synaptic plasticity with time delay. The European Physical Journal Plus, 129(6):1-11, 2014. http://dx.doi.org/10.1140/epjp/i2014-14106-3.

[26] Q. Wang, D. Li and M.Z. Liu. Numerical Hopf bifurcation of Runge-Kutta methods for a class of delay differential equations. Chaos, Solitons $\&$ Fractals, 42(5):3087-3099, 2009. http://dx.doi.org/10.1016/j.chaos.2009.04.008.

[27] Q. Wang and M. Z. Liu. Stability and Neimark-Sacker bifurcation in Runge-Kutta methods for a predator-prey system. International Journal of Computer Mathematics, 86(12):2218-2224, 2009. http://dx.doi.org/10.1080/00207160902787988.

[28] W. Wendi and M. Zhien. Harmless delays for uniform persistence. Journal of Mathematical Analysis and Applications, 158(1):256-268, 1991. http://dx.doi.org/10.1016/0022-247X(91)90281-4.

[29] J. Yan, A. Zhao and W. Yan. Existence and global attractivity of periodic solution for an impulsive delay differential equation with Allee effect. Jour. of Math. Anal. and Appl., 309(2):489-504, 2005. http://dx.doi.org/10.1016/j.jmaa.2004.09.038.

[30] B.G. Zhang and K. Gopalsamy. Global attractivity and oscillations in a periodic delay-logistic equation. Journal of Mathematical Analysis and Applications, 150(1):274-283, 1990. http://dx.doi.org/10.1016/0022-247X(90)90213-Y. 